\title{
ZONAS TRANSVERSALES EN EL DOMINIO AXIAL DE LA CORDILLERA ORIENTAL - BLOQUE YACENTE DE LA FALLA DE SOAPAGA (SÁTIVA SUR - SÁTIVA NORTE, BOYACÁ)
}

\author{
Alvaro Villar ${ }^{1}$; Helmer Alarcón ${ }^{1}$; Giovanny Jiménez ${ }^{1 *}$; Francisco Velandia ${ }^{1}$ \\ DOI: http://dx.doi.org/10.18273/revbol.v39n3-2017001 @c) (1) \\ Forma de citar: Villar, A., Alarcón, H., Jiménez, G., y Velandia, F. 2017. Zonas transversales en el dominio \\ axial de la Cordillera Oriental - bloque yacente de la Falla de Soapaga (Sátiva sur - Sátiva norte, Boyacá). \\ Boletín de Geología, 39(3): 13-23.
}

\section{RESUMEN}

Mediante la cartografía geológica realizada en un sector adyacente al Macizo de Floresta, en la zona axial de la Cordillera Oriental, que incluye la Falla de Soapaga, se definieron tres dominios estructurales según cambios en el estilo del bloque yacente. El dominio norte se caracteriza por presentar un anticlinal regional cortado por fallas inversas de mediano ángulo sub-paralelas y de acomodación de pliegue. En los dominios central y sur se evidencia un sinclinal inclinado que comparte un flanco con el anticlinal regional y presentan vergencia este. Estos dos dominios se encuentran separados por una falla transversal de dirección NWW-SEE. El sistema de fallas de Soapaga se expresa como un abanico imbricado arrastrado con vergencia este. Las fallas transversales pueden sugerir la presencia de una zona de transferencia asociada a anisotropías preexistentes del basamento que han sido reactivadas durante el acortamiento del Cenozoico asociado al levantamiento de la Cordillera Oriental.

Palabras clave: Falla de Soapaga; zona transversal; estilo estructural; Orogenia Andina.

\section{TRANSVERSE ZONES IN THE AXIAL DOMAIN OF THE EASTERN CORDILLERA - FOOTWALL OF THE SOAPAGA FAULT (SÁTIVA SUR - SÁTIVA NORTE, BOYACÁ)}

\begin{abstract}
Three structural domains were interpreted along the Soapaga Fault according to the observed changes of the footwall block, this after the geological mapping of a sector near the Floresta Massif in the Eastern Cordillera axial zone. The northern domain shows a regional anticlinal, which is cut by reverse mid-angle sub-parallel and fold accommodation faults. Is evident that in the in the central and southern domains a tilted syncline shares a limb with the East verging adjacent anticline. These two domains are separated by a NWW transverse zone. The Soapaga fault system is defined in the area as a trailing imbricate fan. Transverse faults may suggest the presence of a transfer zone associated to pre-existing basement anisotropies that have been reactivated during the Eastern Cordillera Cenozoic uplift.
\end{abstract}

Keywords: Soapaga Fault; Transversal zone; structural style; Andean Orogeny.

\footnotetext{
${ }^{1}$ Grupo GIGBA, Escuela de Geología, Universidad Industrial de Santander, Bucaramanga, Santander, Colombia. (*)gjimenezd@gmail.com, helmeralarcon@hotmail.com, alevice.rain@hotmail.com, favelanp@uis.edu.co
} 


\section{INTRODUCCIÓN}

Las fallas transversales, rampas laterales o zonas de transferencia son lineamientos oblicuos a los cinturones plegados que proveen un enlace entre diferentes rampas frontales y transfieren desplazamiento a través del rumbo; constituyendo una zona transversal (Wilson y Stearns, 1958; Harris, 1970; Thomas, 1990). Las fallas transversales se encuentran asociadas en profundidad con anisotropías del basamento, que controlan la deformación en la cubierta sedimentaria durante el acortamiento y ocasionan cambios en el estilo estructural entre áreas adyacentes (Jiménez et al., 2012). Estos lineamientos transversales no están distribuidos aleatoriamente, sino que se extienden atravesando el cinturón plegado sub-perpendicularmente al rumbo regional y sub-paralelamente a la dirección del transporte tectónico (Dahlstrom, 1970; Cook y Thomas, 2009). El control cartográfico permite definir zonas transversales por terminaciones abruptas de fallas a lo largo del rumbo, alineaciones de rampas laterales, cambios en el ángulo y vergencia de estructuras, y cabeceo en los flancos de los pliegues (FIGURA 1) (Thomas, 1990; Thomas y Bayona, 2002).

Los modelos de deformación en el Paleógeno para la parte axial de la Cordillera Oriental continúan siendo objeto de controversia. Estudios previos sugieren una inversión tectónica del orógeno, asociada a fallas normales Mesozoicas, expresado en un estilo estructural de tectónica de piel gruesa (thick-skinned) con cabalgamientos de bajo ángulo a subhorizontales dentro de una tectónica transpresiva (Colletta et al., 1990; Cooper et al., 1995; Toro, 1990; Saylor et al., 2012; Jiménez et al., 2014). Por otro lado, otros autores argumentan que las fallas de Soapaga y Boyacá, en algunos sectores, se comportan como cabalgamientos, pero a su vez están relacionadas regionalmente con estructuras de alto ángulo en profundidad, asociado a fallas que involucran basamento (Kammer, 1999; Taboada et al., 2000; Velandia, 2005; Rodríguez et al., 2009; Tesón et al., 2013).

Este artículo tiene como objetivo definir un estilo estructural y proponer la existencia de una zona transversal en el bloque yacente de la Falla Soapaga, basados en la geología de superficie y la elaboración de cortes geológicos en un sector adyacente al Macizo de Floresta. Adicionalmente, se considera la existencia de una relación genética de dicha zona transversal con estructuras profundas como la Falla de Soapaga, siendo un modelo concordante con aquellos que postulan movimientos en rumbo asociados a la deformación de la cubierta sedimentaria durante el acortamiento de la Cordillera Oriental (FIGURA 2).

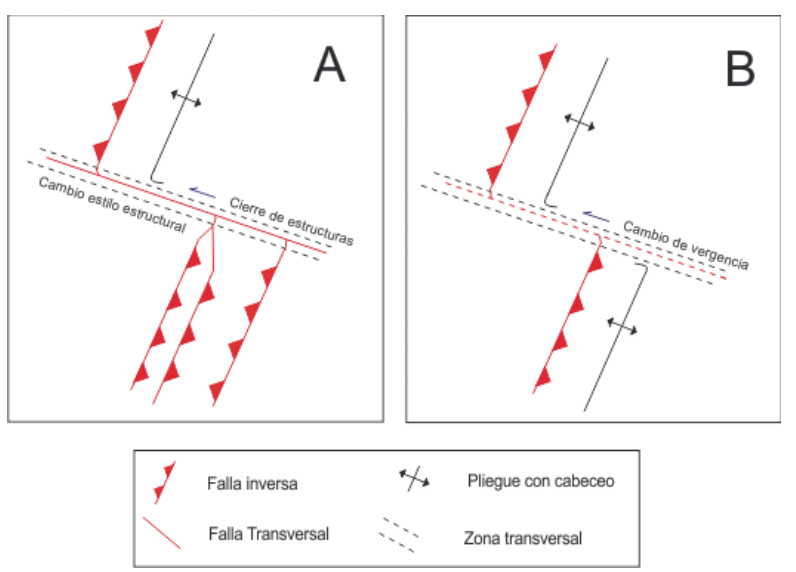

FIGURA 1. A. Zona transversal definida por fallas que generan cambios en el estilo estructural. B. Zona transversal definida por alineación de rampas laterales y cambios de vergencia. Modificado de Jiménez et al. (2012).

\section{MARCO GEOLÓGICO: CORDILLERA ORIENTAL}

Los Andes Colombianos se dividen en tres grandes cinturones montañosos de distintas edades y características geológicas: la Cordillera Occidental y parte de la Cordillera Central al oeste del sistema de fallas de Romeral, compuestas de rocas alóctonas de afinidad oceánica acrecionadas a los 60-65 Ma (Toro et al., 2004; McCourt et al., 1984). La Cordillera Central, compuesta de rocas metamórficas Paleozoicas, y tonalitas-granodioritas Jurásicas y Cretácicas (RestrepoPace, 1992; Villagómez et al., 2011); y la Cordillera Oriental, limitada al oeste por la cuenca intermontana del Valle del Magdalena y al este por la cuenca foreland Llanos infrayacida por el Cratón Amazónico (Horton et al., 2010) (FIGURA 2).

La Cordillera Oriental contiene exposiciones de basamento metamórfico continental de bajo a alto grado, ubicados en el eje axial y tomando el rumbo de la cordillera, como los macizos de Santander y Floresta (Ramirez-Arias et al., 2012; Tesón et al., 2013). Inconformes a ese basamento, se encuentran rocas sedimentarias en cinturones plegados continentales del Jurásico y depósitos marinos del Cretácico, superpuestas por cuencas foreland presevadas en sinclinales del Paleoceno al Oligoceno (Ramirez-Arias et al., 2012). La Cordillera Oriental está conformada por rocas que evidencian distintos procesos de acumulación y levantamiento: la sedimentación marina y no marina durante la extensión Mesozoica evidenciada en la cuenca graben del Triásico - Jurásico y una cuenca backarc durante el Tithoniano-Necomiano hasta el 
Maastrichtiano (Sarmiento-Rojas et al., 2006; Kammer y Sánchez, 2006); y el acortamiento Cenozoico producto de convergencia oblicua de las placas Suramericana, Nazca y Caribe evidenciado en múltiples fases de deformación compresional asociadas a tectónica de piel gruesa y delgada que inicia en el Cretácico tardío (Acosta et al., 2004; Saylor et al., 2012; Bayona et al., 2013) y tiene su mayor actividad en el Plioceno- Pleistoceno.

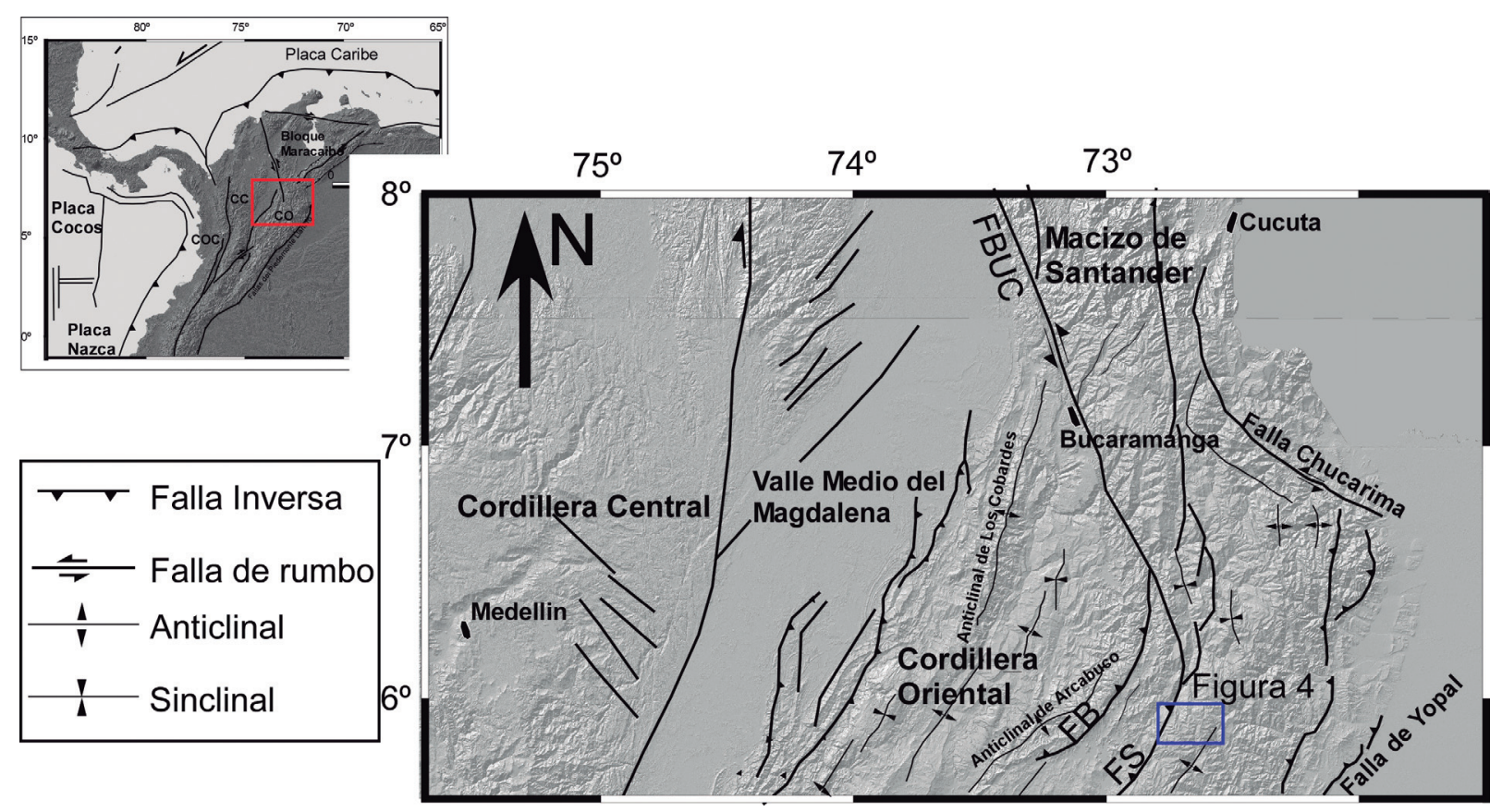

FIGURA 2. Modelo Digital de Elevación (DEM) donde se muestra los límites tectónicos de la Cordillera Oriental (CO) y se enmarca el área de estudio (recuadro azul) ubicado en el bloque yacente de la Falla de Soapaga, adyacente al Macizo de Floresta. $\mathrm{COC}=$ Cordillera Occidental, $\mathrm{CC}=$ Cordillera Central, FB = Falla de Boyacá y FS = Falla de Soapaga.

El acortamiento Cenozoico dio lugar a una cuenca foreland sobre el Valle del Magdalena, Cordillera Oriental y cuenca Llanos (Horton et al., 2010; Mora et al., 2010; Saylor et al., 2011), la cual posteriormente se particionó en pequeños bloques levantados y rellenados con depósitos sin-orogénicos (Bayona et al., 2013), definiendo el levantamiento y actual configuración de la Cordillera Oriental (FIGURA 2) (Dengo y Covey, 1993; Saylor et al., 2012; Mora et al., 2010). Jiménez et al. (2014) sugieren que la curvatura al norte de la Cordillera Oriental está relacionada con un orógeno primario y a movimientos destrales en la zona axial.

La Cordillera Oriental se encuentra definida por un cinturón plegado y fallado asimétrico, de doble vergencia, que presenta una combinación entre deformación de tectónica de piel delgada (thin-skinned) asociada a una compresión este-oeste dentro de una cinemática transpresiva (Bande et al., 2012; Bayona et al., 2013); y una inversión tectónica asociada de fallas normales Mesozoicas (Bayona et al., 2013; Cooper et al., 1995), además de una generación posterior de estructuras de acortamiento de primer orden (Bande et al., 2012).

\section{Dominio axial de la Cordillera Oriental}

El dominio axial de la cordillera consiste en anticlinales apretados y sinclinales amplios relacionados a fallas de doble vergencia, asociadas a estructuras de despegue o pliegues de rompimiento (break-thrust folds) (Mora et al., 2010). El Sistema de fallas Soapaga-Pesca divide el dominio axial en oriental y occidental (Bayona et al., 2013). Las fallas de Soapaga y Boyacá son fallas inversas que actúan como terminación en cola de caballo al sur de la Falla Bucaramanga (Toro, 1990; Dengo y Covey, 1993; Kammer y Sánchez, 2006; Velandia, 2005; Tesón et al., 2013).

Las fallas de cabagamiento se dividen en intervalos de $10-40 \mathrm{~km}$ y presentan altos de basamento que controlan la sedimentación de las subcuencas adyacentes (Kammer y Sánchez, 2006), además, se caracterizan por una segmentación longitudinal de decenas de kilómetros y sus expresiones estructurales se manifiestan en sinclinales marginales en los bloques levantados y un basculamiento del bloque yacente hacia el bloque colgante (Kammer, 1996). 


\section{Estratigrafía}

Para este estudio se utilizó la nomenclatura propuesta para las planchas de 152-Soatá (Vargas et al., 1987) y 172-Paz del Rio (Ulloa et al., 1998). La secuencia sedimentaria corresponde a la región del Macizo de Floresta y al bloque yacente de la Falla de Soapaga, con unidades que varían en edad desde el Paleozoico al Paleógeno (FIGURA 3). Las rocas ígneas y metamórficas que conforman el basamento cristalino del Macizo de Floresta datan del Paleozoico inferior, y son principalmente esquistos micáceos cortados por intrusiones graníticas, y suprayacidos por unidades no metamorfizadas como las formaciones Tíbet y Floresta del Devónico y Cuche del Carbonífero (Mojica y Villarroel, 1984; Vargas et al., 1987) (FIGURA 3). La Formación Girón está compuesta por areniscas y conglomerados continentales rojizos de edad Jurásico Superior (Royero y Clavijo, 2001) y se encuentra en disposición discordante con las metamorfitas pre-Mesozoicas (Mojica y Villarroel, 1984). Su espesor varía entre $1 \mathrm{~km}$, cercano a las fallas de Boyacá y Soapaga, a $300 \mathrm{~m}$ hacia el oeste, evidenciando un rápido adelgazamiento (Kammer y Sánchez, 2006; Saylor et al., 2012) (FIGURA 3).

Las unidades marinas del Cretácico están representadas por el Grupo Uribante (Fabre, 1985), discordante sobre la Formación Girón (Cooper et al., 1995), el Grupo Guadalupe y parte de la Formación Guaduas. La secuencia empieza con los shales y calizas de la Formación Tibú-Mercedes (Vargas et al., 1987) de edad Aptiano-Albiano (Ulloa et al., 1998), seguido de las cuarzoarenitas glauconíticas de la Formación Aguardiente de edad Albiano (Ulloa et al., 1998; Caballero y Sierra, 1991) y los shales y calizas fosilíferas de la Formación Capacho (Caballero y Sierra, 1991) de edad TuronianoCenomaniano (Ulloa et al., 1998) (FIGURA 3). El Grupo Guadalupe está compuesto de una sucesión de areniscas granocrecientes, intercaladas con limolitas y lodolitas negras macizas, evidenciadas en las formaciones Labor-Tierna, Pinos y Plaeners (Saylor et al., 2012) del Coniaciano-Santoniano (Ulloa et al., 1998). La Formación Guaduas representa la transición de ambientes marinos a continentales (Cooper et al., 1995) coincidiendo con el límite Cretácico-Paleógeno; está compuesta de capas de arcillas negras carbonosas, intercaladas con areniscas y mantos de carbón (Sarmiento, 1992; Guerrero y Sarmiento, 1996). Esta unidad data del Maastrichtiano superior al Paleoceno inferior (Pardo y Jaramillo, 2014) (FIGURA 3).

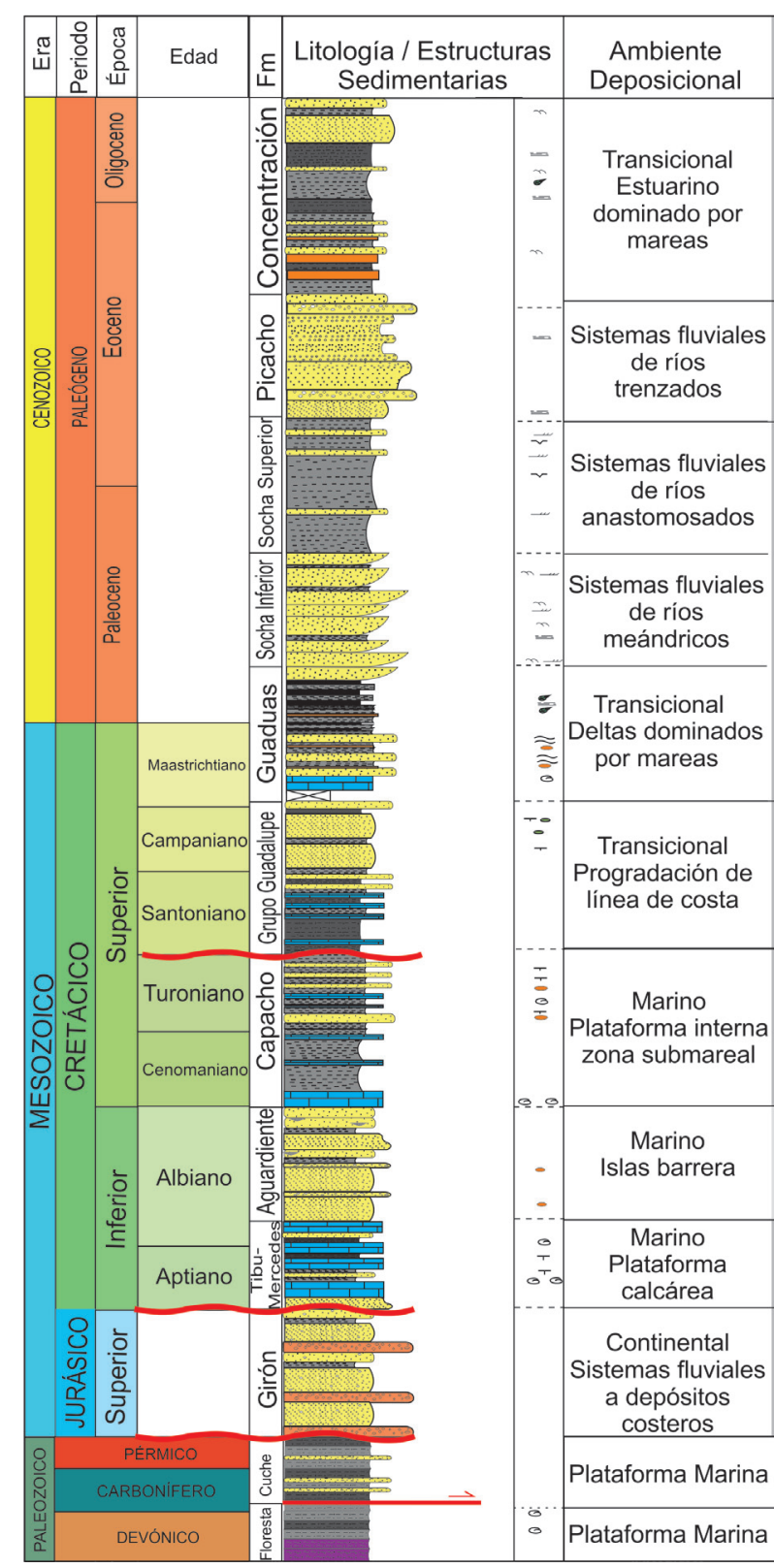

FIGURA 3. Columna estratigráfica generalizada de las unidades presentes desde el Devónico hasta el Oligoceno. Las líneas rojas irregulares corresponden a disconformidades regionales y límites de secuencia. Modificado de Alarcón y Villar (2016).

Los estratos Cenozoicos que suprayacen la secuencia Cretácica se encuentran de forma concordante y corresponden a la Formación Socha Inferior y Socha Superior (Guerrero y Sarmiento, 1996), una sucesión de cuarzoarenitas lenticulares con evidentes estructuras de flujo y arcillolitas y lodolitas con rastros de materia orgánica, que corresponden a depósitos fluviales desde meandriformes a anastomosados (Guerrero y Sarmiento, 1996; Saylor et al., 2011), de edad Paleoceno Temprano 
a Tardío (Pardo y Jaramillo, 2014) (FIGURA 3). Las unidades más jóvenes corresponden a las formaciones Picacho y Concentración del Eoceno medio a Oligoceno inferior (Pardo y Jaramillo, 2014). En el sector de Sátiva sur, la Formación Picacho se interpreta como depósitos fluviales de tipo trenzado en capas de areniscas conglomeráticas y conglomerados cuarzosos (Saylor et al., 2011; Bayona et al., 2013), mientras que la Formación Concentración corresponde a capas de areniscas de grano fino intercaladas con lodolitas de un ambiente estuarino a deltaico (Saylor et al., 2011; Bayona et al., 2013) (FIGURA 3).

\section{MÉTODOS}

Se realizó la cartografía geológica a escala 1:25.000 de una zona correspondiente al bloque yacente de la
Falla de Soapaga en el sector de Sátiva Sur, donde se caracterizaron sedimentológicamente las unidades del Cretácico y Paleógeno aflorantes. Se llevó a cabo la adquisición de datos estructurales (planos de estratificación, estrías de falla y diaclasas). Los datos colectados fueron corregidos con base en la declinación magnética para el área, obtenida de la National Oceanic and Atmospheric Administration (NOAA) la cual corresponde a $8^{\circ}$ para el 2016. Se integraron los datos a disposición con la cartografía propuesta en la plancha 152-Soatá a escala 1:100.000 (Vargas et al., 1987), para obtener un mapa regional a partir del cual se elaboraron tres cortes geológicos, asumiendo un flujo paralelo a la falla y basados en el método de Kink (Dutch, 2000) para la construcción de secciones estructurales. Los cortes siguieron una orientación preferencial perpendicular a la estratificación (FIGURA 4).

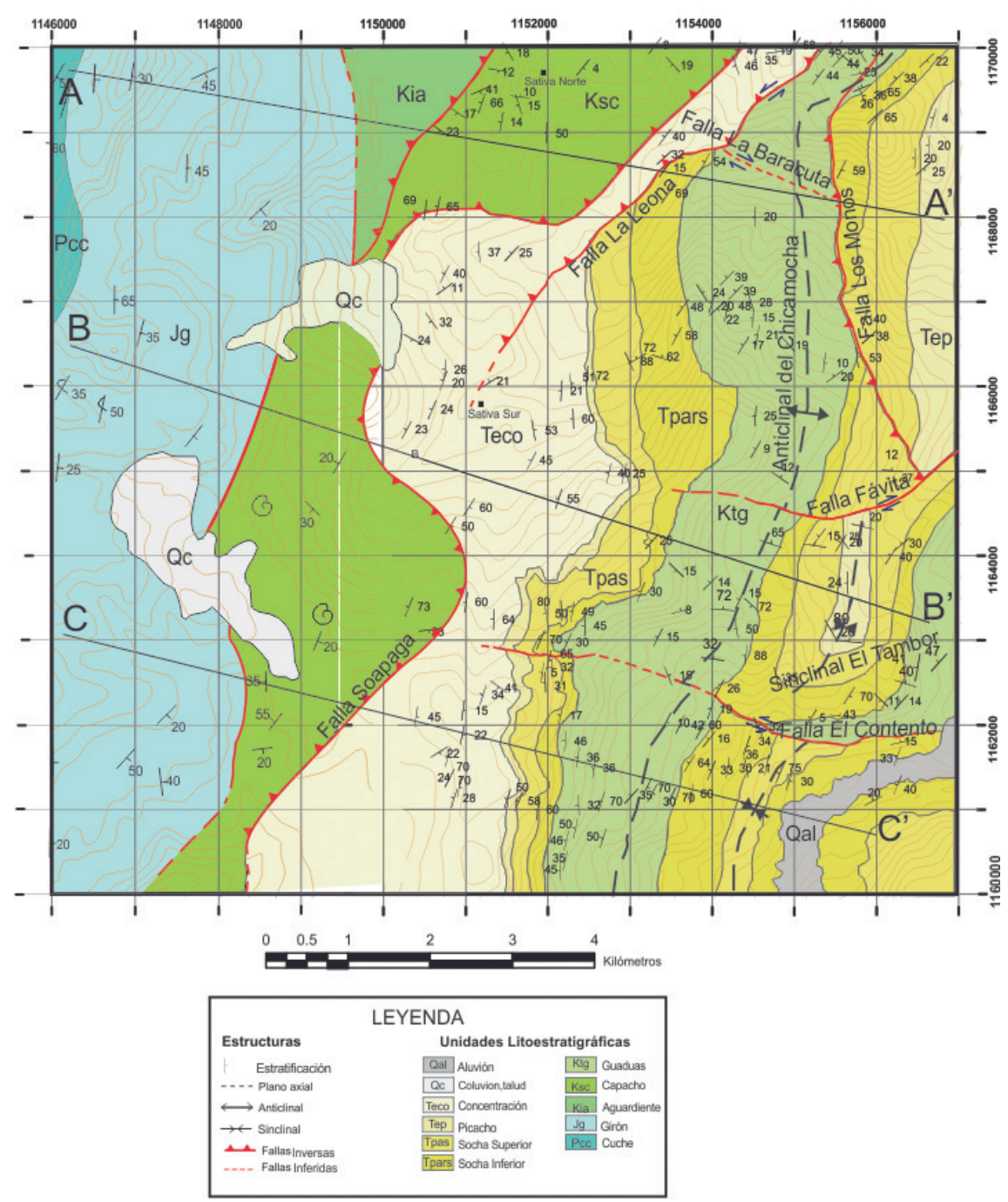

FIGURA 4. Mapa geológico del área de estudio y zonas adyacentes. Tomado y modificado de Vargas et al. (1987) y Alarcón y Villar (2016). 


\section{RESULTADOS}

\section{Cartografía}

Integrando la cartografía realizada en la zona a escala 1:25.000 con la información bibliográfica disponible, se reconocieron unidades estratigráficas desde el Carbonífero al Oligoceno. Estructuralmente, el área está dominada por la presencia de fallas inversas y pliegues (anticlinales y sinclinales). La Falla de Soapaga, es una falla inversa regional, con una orientación NE-SW, que en el sector cartografíado pone en contacto rocas Cretácicas con la Formación Concentración del Oligoceno. El Anticlinal del Chicamocha corresponde a un pliegue abierto en cuyo flanco occidental aflora la secuencia paleógena desde la Formación Guaduas hasta la Formación Concentración (FIGURA 4).

La cartografía realizada permite dividir la zona en tres dominios estructurales: norte, central y sur. Esto con base en las diferencias del estilo estructural del bloque yacente de la Falla de Soapaga (FIGURA 4). El dominio norte se caracteriza porque el Anticlinal del Chicamocha presenta un rumbo NE-SW y vergencia Occidente y su flanco oriental se encuentra cortado por una falla inversa con transporte al Oriente (FIGURA 4). Así mismo, se encuentra una falla inversa por acomodación de pliegue adyacente al occidente, que se desprende del núcleo del Sinclinal de Bura, cabalgando rocas de la Formación Concentración sobre unidades más antiguas de la Formación Guaduas.
El dominio central está enmarcado por dos fallas transversales con rumbo SWW-NEE y NWW-SEE, las cuales limitan y actúan como cierre de un sinclinal apretado y volcado, llamado El Tambor (Alarcón y Villar, 2016); donde aflora la Formación Picacho en el núcleo. En este dominio, el Anticlinal del Chicamocha tiene rumbo que varía entre N-S y NE-SW y vergencia Oriente (FIGURA 4).

En el dominio sur, el Anticlinal del Chicamocha presenta un rumbo SSW-NNE con vergencia Oriente; en la parte oriental se observa como rasgo característico el cambio en el ángulo de buzamiento entre el flanco oriental, con inclinaciones $<30^{\circ}$, y el flanco occidental del Sinclinal El Tambor. En el núcleo del sinclinal aflora la Formación Socha Superior (FIGURA 4).

\section{Geología estructural}

Se realizan tres cortes geológicos en dirección NW-SE que permiten interpretar la geometría en profundidad y un estilo estructural de sistemas de cabalgamientos por tectónica de piel delgada, expresado en fallas en abanico contraccional imbricado y arrastrado con vergencia Este.

El corte A-A' se ubica en la parte más septentrional de la zona y corresponde al dominio norte. Hacia el Occidente se observan la Falla de Soapaga y Bucaramanga (Toro, 1990) que se enlazan en cabalgamientos emergentes y sub-paralelos asociados a un abanico imbricado arrastrado con vergencia Oriente (FIGURA 5).

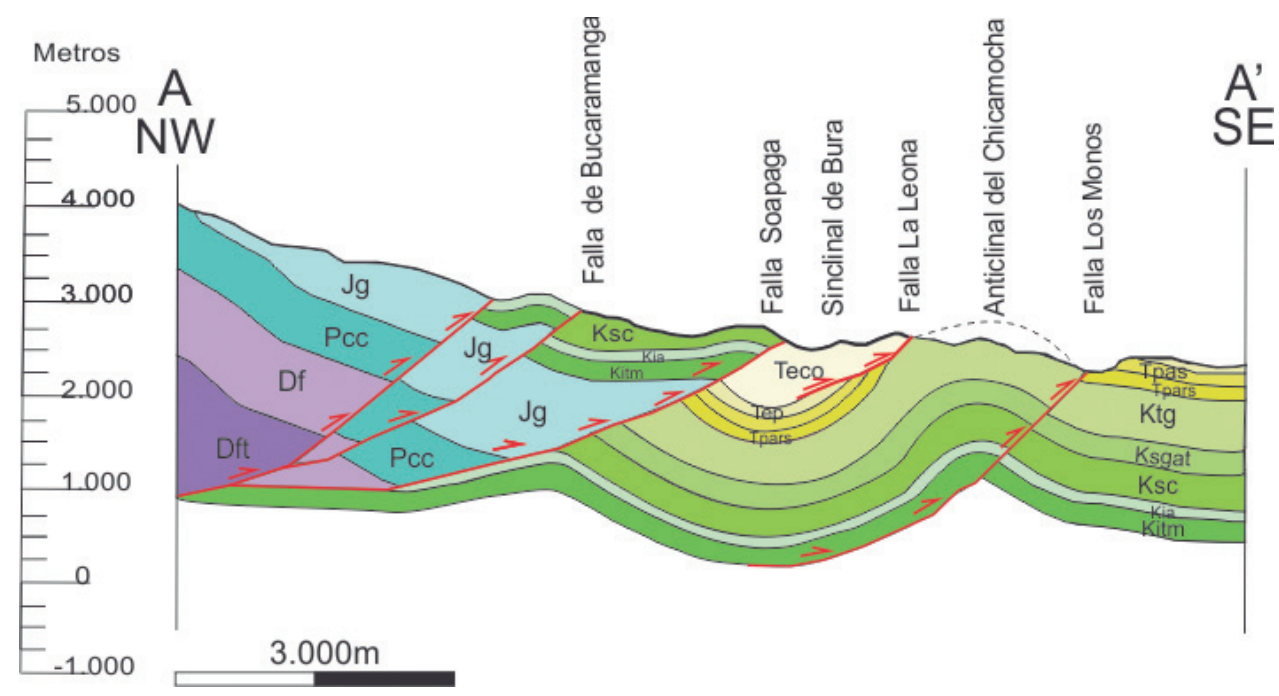

FIGURA 5. Corte geológico A-A' ubicado en el dominio norte de la zona. En los tres dominios estructurales, la Falla de Soapaga se presenta con rumbo SW-NE y alta sinuosidad en superficie indicando un ángulo bajo de buzamiento. El bloque colgante de la Falla de Soapaga exhibe rocas cretácicas de las formaciones Capacho y Aguardiente, en contacto con la Formación Concentración, y más al oeste las formaciones Girón y Cuche (FIGURA 4). 
En el bloque yacente de la Falla Soapaga, se evidencia una falla por acomodación de pliegue del tipo fuera del sinclinal (Mitra, 2002), llamada Falla La Leona (Alarcón y Villar, 2016). Esta se trata de una estructura de bajo ángulo que se desprende del núcleo del Sinclinal de Bura, cabalgando rocas más jóvenes de la Formación Concentración sobre más antiguas de la Formación Guaduas. En el dominio norte, uno de los rasgos estructurales más importantes es el Anticlinal del Chicamocha, que se presenta como un pliegue abierto con vergencia Occidente. Este presenta ángulos de buzamiento de $40^{\circ}$ a $60^{\circ}$ en su flanco occidental. El flanco oriental se encuentra cortado por la Falla Los Monos (Alarcón y Villar, 2016), una estructura inversa con vergencia Oriente, asociada a un despegue que en profundidad se genera en unidades incompetentes del Cretácico y que se expresa como una rampa de mediano ángulo (FIGURA 5).

El corte B-B' fue trazado a lo largo del dominio central de la zona y atraviesa perpendicularmente el sistema de abanicos imbricados de la Falla de Soapaga, que ponen en contacto la Formación Capacho con la Formación Concentración. En el bloque yacente de la Falla de Soapaga, el Anticlinal del Chicamocha presenta un cambio de vergencia hacia el Oriente, conservando el ángulo abierto entre flancos, pero presentando mayores inclinaciones en su flanco oriental. Por otra parte, el Sinclinal El Tambor, se evidencia como un pliegue cerrado y volcado (inclinado), con su flanco occidental invertido con inclinaciones de $40^{\circ}$ hacia el mismo sentido en ambos flancos (FIGURA 6).

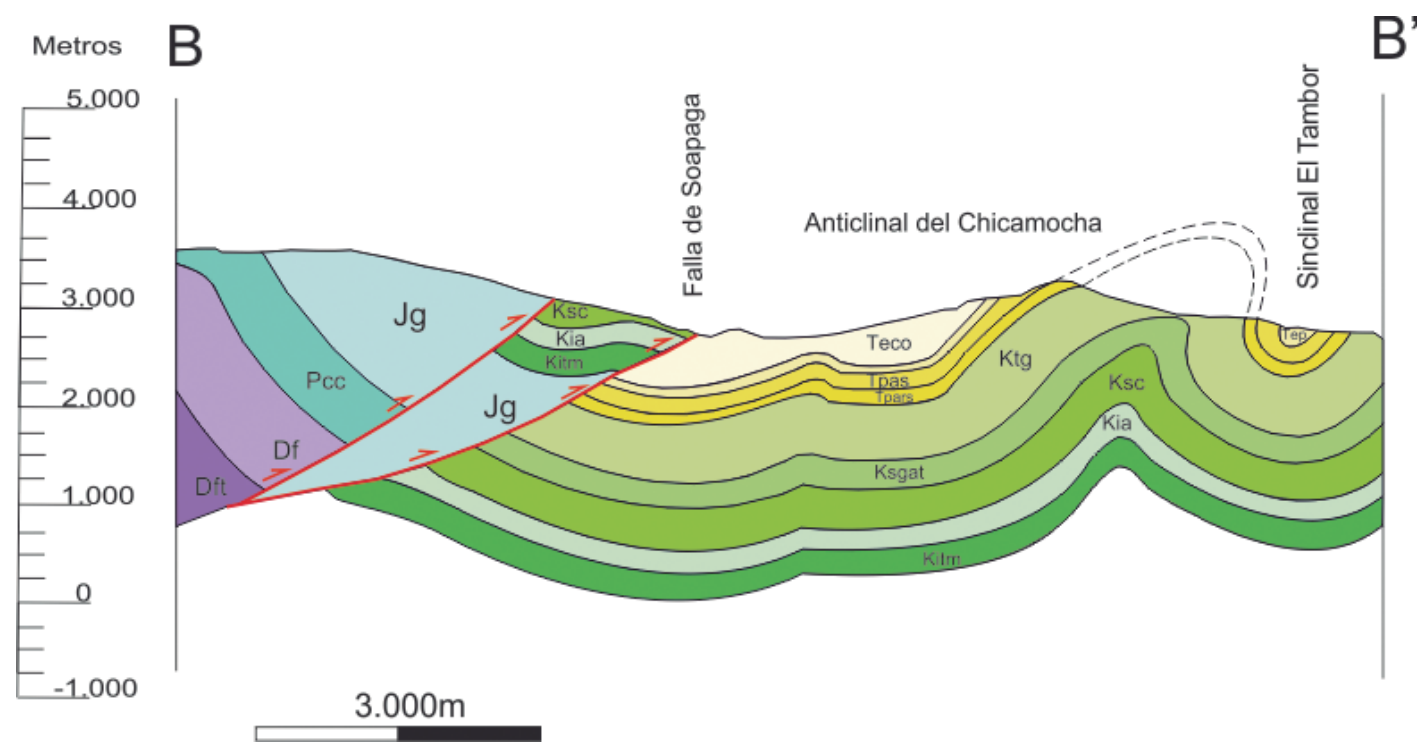

FIGURA 6. Corte geológico B-B' ubicado en el dominio central de la zona.

El corte C-C' está ubicado en el dominio sur de la zona. En esta sección, se presenta nuevamente la Falla de Soapaga, localizada en el flanco occidental del Sinclinal de Bura. El bloque yacente de la Falla de Soapaga, se caracteriza por la ausencia de las fallas identificadas hacia el norte del área (Falla La Leona, Falla Los Monos y las fallas transversales) y se encuentra el Anticlinal del Chicamocha, definido como un pliegue abierto con vergencia Oriente. Así mismo, el Sinclinal El Tambor refleja un gran cambio en el ángulo entre sus flancos, expresándose como un pliegue abierto con ángulos de buzamiento menores a $20^{\circ}$ en su flanco oriental (FIGURA 7).

\section{DISCUSIÓN}

En la Cordillera Oriental, las fallas inversas que involucran basamento, se caracterizan por una segmentación longitudinal de decenas de kilómetros y son interpretadas como fallas normales reactivadas (Montes et al., 2008; Bayona et al., 2008; Horton et al., 2010). La segmentación de estas estructuras conforma el modelo de crecimiento de la falla, combinando acumulación de desplazamiento en buzamiento con expansión de fallas longitudinales (Kammer y Sánchez, 2006). Toro (1990) expresa un modelo de abanicos imbricados donde evidencia el cierre de la Falla de Bucaramanga contra la 
Falla de Soapaga en el área de estudio. Las expresiones estructurales de estas fallas regionales, como la Falla de Soapaga, se manifiestan por la presencia de cinturones plegados mesozoicos, suprayacidos por depósitos del Paleoceno al Oligoceno, preservados en sinclinales amplios y anticlinales apretados (Kammer y Sanchez, 2006; Ramirez-Arias et al., 2012; Bayona et al., 2013), como el Sinclinal de Bura y el Anticlinal del Chicamocha, ubicados en el bloque yacente de la Falla de Soapaga (FIGURAS 4, 5 y 6 ).

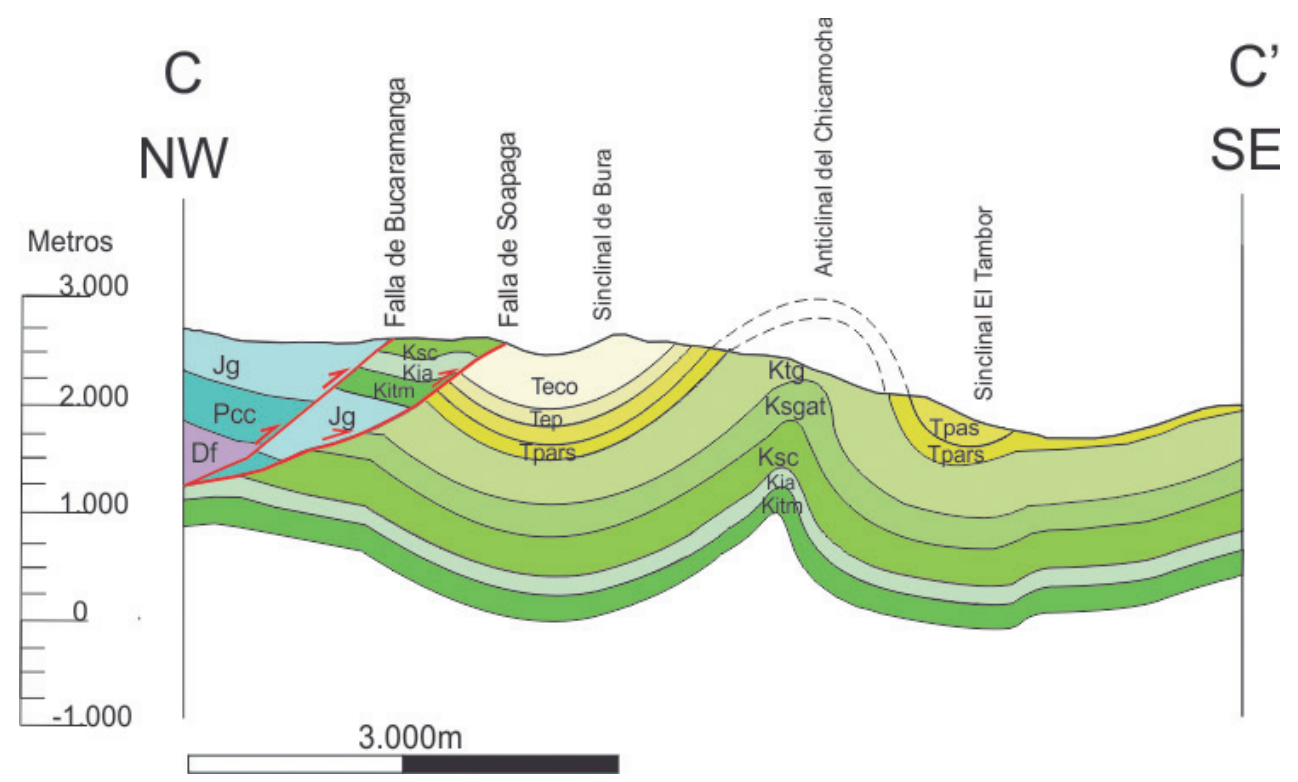

FIGURA 7. Corte geológico C-C' ubicado en el dominio sur de la zona.

El bloque yacente de la Falla Soapaga evidencia una serie de fallas transversales NWW-SEE y NEE-SWW, la Falla Fávita y la Falla El Contento (FIGURA 4). Algunos lineamientos transversales con direcciones similares son reportados por Reyes (2001) y Velandia (2005), en estudios cercanos a la Falla de Soapaga. La Falla Fávita se define con un rumbo NEE-SWW, la cual se caracteriza por controlar los cambios de vergencia en el Anticlinal del Chicamocha (FIGURAS 4 y 5), además actúa como cierre de fallas (e.g Falla Los Monos) (FIGURA 4). La Falla El Contento tiene una dirección NWW-SEE, se encuentra cortando estructuras regionales longitudinales como el Anticlinal del Chicamocha y se presenta como cierre con inmersión del Sinclinal El Tambor (FIGURA 4).

Estos lineamientos transversales se interpretan como anisotropías preexistentes del basamento que controlan la geometría de los pliegues y la vergencia de las estructuras (Thomas y Bayona, 2002; Cook y Thomas, 2009; Jiménez et al., 2012; García y Jiménez, 2016). Los cambios laterales de espesores estratigráficos a lo largo del rumbo, como la Falla Fávita, se asocian en secuencias de rift invertidas (Kwon y Mitra, 2012), a rampas laterales. Los cambios de vergencia de las estructuras, las terminaciones abruptas de fallas y pliegues, los cambios en el estilo estructural y la transferencia y el cambio de desplazamiento a lo largo del rumbo, permiten definir una zona transversal para el área de estudio. Estas zonas transversales fueron estructuras formadas por adelgazamiento cortical durante la evolución del graben Mesozoico en la Cordillera Oriental reactivadas con una componente en rumbo subparalelo al tensor regional durante la pase principal de la deformación Andina. Según Taboada et al. (2000) la reactivación ocurrió bajo un régimen compresivo homogéneo con dirección E-SE, por otro lado según Toro y Osorio (2005) el vector de convergencia de la placa Nazca se particiona y define al norte de los $5^{\circ} \mathrm{N}$ un vector con dirección WSW- ENE. Las zonas trasversales definidas además, afectan la evolución de las estructuras compresionales subsecuentes durante la inversión de la cuenca (Konstantinovskaya et al., 2007), como el relleno sedimentario sin-tectónico cenozoico del bloque yacente de la Falla de Soapaga.

\section{CONCLUSIONES}

El relleno sin-tectónico de las unidades paleógenas en la Cordillera Oriental, como la secuencia sedimentaria encontrada en el bloque yacente de la Falla de Soapaga, 
presenta cambios en el estilo estructural debido a la acción de fallas regionales reactivadas como la Falla de Soapaga, y a zonas transversales que controlan la extensión y la geometría de fallas y pliegues regionales. El estilo estructural del bloque yacente de la Falla de Soapaga se basa en cabalgamientos por tectónica de piel delgada expresada en un abanico imbricado arrastrado con vergencia este, que generan anticlinales abiertos y sinclinales apretados con cambios de vergencia.

Los lineamientos de dirección NEE-SWW y NWWSEE controlan la vergencia de las estructuras y actúan como cierre de pliegues y fallas, definiendo una zona transversal. Esta zona de transferencia se encuentra asociada a anisotropías preexistentes del basamento heredadas del antiguo graben Mesozoico en la Cordillera Oriental.

\section{AGRADECIMIENTOS}

Los autores queremos agradecer a los estudiantes de los cursos de Campo I de la Universidad Industrial de Santander quienes participaron activamente en el reconocimiento de la zona de estudio. Además los autores agradecen a los revisores José Fernando Duque y Felipe Lamus por sus valiosos comentarios y sugerencias en el manuscrito.

\section{REFERENCIAS}

Acosta, J., Lonergan, L., and Coward, M.P. 2004. Oblique transpression in the western thrust front of the Colombian Eastern Cordillera. Journal of South American Earth Sciences, 17(3): 181-194.

Alarcón, H., y Villar, A. 2016. Integración de datos estratigráficos y estructurales de las unidades Paleógenas presentes en el bloque yacente de la Falla de Soapaga. Tesis, Universidad Industrial de Santander, Bucaramanga. 149p.

Bande, A., Horton, B.K., Ramírez, J.C., Mora, A., Parra, M., and Stockli, D.F. 2012. Clastic deposition, provenance, and sequence of Andean thrusting in the frontal Eastern Cordillera and Llanos foreland basin of Colombia. Geological Society of America Bulletin, 124(1-2): 59-76.

Bayona, G., Cortés, M., Jaramillo, C., Ojeda, G., Aristizabal, J.J., and Reyes-Harker, A. 2008. An integrated analysis of an orogen-sedimentary basin pair: Latest Cretaceous-Cenozoic evolution of the linked Eastern Cordillera orogen and the Llanos foreland basin of Colombia. GSA Bulletin, 120(9-10): 1171-1197.
Bayona, G., Cardona, A., Jaramillo, C., Mora, A., Montes, C., Caballero, V., Mahecha, H., Lamus, F., Montenegro, O., Jimenez, G., Mesa, A., and Valencia, V. 2013. Onset of fault reactivation in the Eastern Cordillera of Colombia and proximal Llanos Basin; response to Caribbean-South American convergence in early Palaeogene time. Geological Society, London, Special Publications, 377(1): 285-314.

Caballero, V., y Sierra, H. 1991. Estratigrafía del Cretácico en el Área de San Andrés, Santander. Tesis, Universidad Industrial de Santander, Bucaramanga. 120p.

Colletta, B., Hebrard, F., Letouzey, J., Werner, P., and Rudkiewicz, J. 1990. Tectonic style and crustal structure of the Eastern Cordillera (Colombia) from a balanced cross-section. In: Letouzey, J. (Ed.). Petroleum and tectonics in mobile belts. Editions Technip. Paris, pp. 81-100.

Cook, B.S., and Thomas, W.A. 2009. Superposed lateral ramps in the Pell City thrust sheet, Appalachian thrust belt, Alabama. Journal of Structural Geology, 31(9): 941-949.

Cooper, M., Addison, F., Alvarez, R., Coral, M., Graham, R., Hayward, A., Howe, S., Martínez, J., Naar, J., Peñas, R., Pulham, J., and Taborda, A. 1995. Basin development and tectonic history of the Llanos Basin, Eastern Cordillera, and Middle Magdalena Valley, Colombia. AAPG Bulletin, 79(10): 1421-1443.

Dahlstrom, C.D. 1970. Structural geology in the eastern margin of the Canadian Rocky Mountains. CSPG Bulletin, 18(3): 332-406.

Dengo, C.A., and Covey, M.C. 1993. Structure of the Eastern Cordillera of Colombia: Implications for trap styles and regional tectonics. AAPG Bulletin, 77(8): 1315-1337.

Dutch, S. 2000. Construct a Fold Cross-Section Using the Kink Method. University of Winconsin-GreenBay. Consultado el 15 de enero de 2016. https://www.uwgb. edu/dutchs/STRUCTGE/SL162KinkMethod.HTM

Fabre, A. 1985. Dinámica de la sedimentación Cretácica en la región de la Sierra Nevada del Cocuy (Cordillera Oriental de Colombia). En: Etayo-Serna, F., y LaverdeMontaño, F. (Eds.). Proyecto Cretácico, contribuciones. Publicaciones Geológicas Especiales, 16: pp. 1-20.

García, H., and Jiménez, G. 2016. Transverse zones controlling the structural evolution of the Zipaquira 
Anticline (Eastern Cordillera, Colombia): Regional implications. Journal of South American Earth Sciences, 69: 243-258.

Guerrero, J., y Sarmiento, G. 1996. Estratigrafía física, palinológica, sedimentológica y secuencial del Cretácico Superior y Paleoceno del Piedemonte Llanero: Implicaciones en exploración petrolera. Geología Colombiana, 20: 3-66.

Harris, L.D. 1970. Details of thin-skinned tectonics in parts of Valley and Ridge and Cumberland Plateau provinces of the southern Appalachians. In: Fisher, G.W., Pettijohn, F.J., Reed, J.C., and Weaver, K.N. (Eds.). Studies of Appalachian Geology: Central and Southern. Interscience. New York, pp. 161-173.

Horton, B.K., Saylor, J.E., Nie, J., Mora, A., Parra, M., Reyes-Harker, A., and Stockli, D.F. 2010. Linking sedimentation in the northern Andes to basement configuration, Mesozoic extension, and Cenozoic shortening: Evidence from detrital zircon $\mathrm{U}-\mathrm{Pb}$ ages, Eastern Cordillera, Colombia. Geological Society of America Bulletin, 122(9-10): 1423-1442.

Jiménez, G., Rico, J., Bayona, G., Montes, C., Rosero, A., and Sierra, D. 2012. Analysis of curved folds and fault/fold terminations in the southern Upper Magdalena Valley of Colombia. Journal of South American Earth Sciences, 39: 184-201.

Jiménez, G., Speranza, F., Faccenna, C., Bayona, G., and Mora, A. 2014. Paleomagnetism and magnetic fabric of the Eastern Cordillera of Colombia: Evidence for oblique convergence and nonrotational reactivation of a Mesozoic intracontinental rift. Tectonics, 33(11): 2233-2260.

Kammer, A. 1996. Estructuras y deformaciones del borde oriental del Macizo de Floresta. Geología Colombiana, 21: 65-80.

Kammer, A. 1999. Observaciones acerca de un origen transpresivo de la Cordillera Oriental. Geología Colombiana, 24: 29-53.

Kammer, A., and Sánchez, J. 2006. Early Jurassic rift structures associated with the Soapaga and Boyacá faults of the Eastern Cordillera, Colombia: Sedimentological inferences and regional implications. Journal of South American Earth Sciences, 21(4): 412-422.

Konstantinovskaya, E.A., Harris, L.B., Poulin, J., and Ivanov, G.M. 2007. Transfer zones and fault reactivation in inverted rift basins: Insights from physical modelling. Tectonophysics, 441(1-4): 1-26.

Kwon, S., and Mitra, G. 2012. An alternative interpretation for the map expression of "abrupt" changes in lateral stratigraphic level near transverse zones in fold-thrust belts. Geoscience Frontiers, 3(4): 401-406.

McCourt, W.J., Aspden, J.A., and Brook, M. 1984. New geological and geochronological data from the Colombian Andes: Continental growth by multiple accretion. Journal of the Geological Society, 141(5): 831-845.

Mitra, S. 2002. Fold-accommodation faults. AAPG Bulletin, 86(4): 671-694.

Mojica, J., y Villarroel, C. 1984. Contribución al conocimiento de las unidades Paleozoicas del área de Floresta (Cordillera Oriental Colombiana; Departamento de Boyacá) y en especial al de la Formación Cuche. Geología Colombiana, 13: 55-79.

Montes, L.A., Hernández, O., Kammer, A., Guerrero, J., Jaramillo, J.M., Escandón, C., Aguilera, R., Téllez, J., Alexander, G., Rodríguez, D.M., Medina, E., Piraquive, A., del Valle, A., and Cerón, M. 2008. Prospectivity of the Eastern Cordillera Basin, Colombia. Agencia Nacional de Hidrocarburos, Colombia.

Mora, A., Horton, B.K., Mesa, A., Rubiano, J., Ketcham, R.A., Parra, M., and Stockli, D.F. 2010. Migration of Cenozoic deformation in the Eastern Cordillera of Colombia interpreted from fission track results and structural relationships: Implications for petroleum systems. AAPG Bulletin, 94(10): 1543-1580.

Pardo, A., y Jaramillo, C. 2014. Palinología y paleoambientes de los depósitos Paleógenos del sector central de la Cordillera Oriental Colombiana: 35 Millones de años de historia de la vegetación neotropical. Colombia Diversidad Biótica XI. Universidad Nacional de Colombia.

Ramirez-Arias, J. C., Mora, A., Rubiano, J., Duddy, I., Parra, M., Moreno, N. and Casallas, W. 2012. The asymmetric evolution of the Colombian Eastern Cordillera. Tectonic inheritance or climatic forcing? New evidence from thermochronology and sedimentology. Journal of South American Earth Sciences, 39: 112-137.

Restrepo-Pace, P.A. 1992. Petrotectonic characterization of the Central Andean Terrane, Colombia. Journal of South American Earth Sciences, 5(1): 97-116. 
Reyes, I. 2001. Neotectónica del corredor industrial de Boyacá, Colombia. VIII Congreso Colombiano de Geología. Memorias. Manizales.

Rodríguez, D.M., Hernández, O., y Kammer, A. 2009. Modelo estructural de la Falla de Soapaga a partir de correlación espectral de anomalías gravimétricas y magnéticas en la Cordillera Oriental, Colombia. Boletín de Geología, 31(2): 35-50.

Royero, J.M., y Clavijo, J. 2001. Memoria Explicativa Mapa Geológico Generalizado Departamento de Santander, Escala 1: 400.00. INGEOMINAS, Bogotá.

Sarmiento, G. 1992. Estratigrafía y medios de depósito de la Formación Guaduas. Boletín Geológico, 32(1-3): 3-44.

Sarmiento-Rojas, L.F., Van Wess, J.D., and Cloetingh, S. 2006. Mesozoic transtensional basin history of the Eastern Cordillera, Colombian Andes: Inferences from tectonic models. Journal of South American Earth Sciences, 21(4): 383-411.

Saylor, J.E., Horton, B.K., Nie, J., Corredor, J., and Mora, A. 2011. Evaluating foreland basin partitioning in the northern Andes using Cenozoic fill of the Floresta basin, Eastern Cordillera, Colombia. Basin Research, 23(4): 377-402.

Saylor, J.E., Horton, B.K., Stockli, D.F., Mora, A., and Corredor, J. 2012. Structural and thermochronological evidence for Paleogene basement-involved shortening in the axial Eastern Cordillera, Colombia. Journal of South American Earth Sciences, 39: 202-215.

Taboada, A., Rivera, L., Fuenzalida, A., Cisternas, A., Philip, H., Bijwaard, H., Olaya, J., and Rivera, C. 2000. Geodynamics of the northern Andes: Subductions and intracontinental deformation (Colombia). Tectonics, 19(5): 787-813.

Tesón, E., Mora, A., Silva, A., Namson, J., Teixell, A., Castellanos, J., and Valencia, V. 2013. Relationship of Mesozoic graben development, stress, shortening magnitude, and structural style in the Eastern Cordillera of the Colombian Andes. Geological Society, London, Special Publications, 377(1): 257-283.

Thomas, W.A. 1990. Controls on locations of transverse zones in thrust belts. Eclogae Geologicae Helvetiae, 83(3): 727-744.

Thomas, W.A., and Bayona, G. 2002. Palinspastic restoration of the Anniston transverse zone in the
Appalachian thrust belt, Alabama. Journal of Structural Geology, 24(4): 797-826.

Toro, J. 1990. The termination of the Bucaramanga fault in the Cordillera Oriental, Colombia. M.Sc. Thesis, The University of Arizona, Arizona. 60p.

Toro, J., Roure, F., Bordas-Le Floch, N., Le CornecLance, S., and Sassi, W. 2004. Thermal and kinematic evolution of the Eastern Cordillera fold and thrust belt, Colombia. Deformation, fluid flow, and reservoir appraisal in foreland fold and thrust belts. AAPG Hedberg Series, 1: 79-115.

Toro, A., y Osorio, J. 2005. Determinación de los tensores de esfuerzos actuales para el segmento norte de los Andes calculados a partir de mecanismos focales de sismos mayores. Boletín de Geología, 27(44): 14-24.

Ulloa, C., Guerra, A., y Escovar, R. 1998. Geología Plancha 172 - Paz de Río. Escala 1:100.000. INGEOMINAS. Bogotá.

Vargas, R., Arias, A., Jaramillo, L., y Téllez, N. 1987. Geología Plancha 152 - Soatá. Escala 1:100.000. INGEOMINAS. Bogotá.

Velandia, F. 2005. Interpretación de transcurrencia de las fallas Soapaga y Boyacá a partir de imágenes Landsat TM. Boletín de Geología, 27(1): 81-94.

Villagómez, D., Spikings, R., Magna, T., Kammer, A., Winkler, W., and Beltrán, A. 2011. Geochronology, geochemistry and tectonic evolution of the Western and Central cordilleras of Colombia. Lithos, 125(3): 875-896.

Wilson, C.W., and Stearns, R.G. 1958. Structure of the Cumberland Plateau, tennessee. Geological Society of America Bulletin, 69(10): 1283-1296.

Trabajo recibido: mayo 15 de 2017

Trabajo aceptado: julio 24 de 2017 